\title{
Functional and Physical Properties of Instant Beverage Powder Made From Two Different Varieties of Pearl Millet
}

\author{
A.O. OBILANA ${ }^{1, *}$, B. ODHAV ${ }^{2}$, V.A. JIDEANI ${ }^{1}$ \\ ${ }^{1}$ Food Technology Department, Cape Peninsula University of Technology, Cape Town, South Africa \\ ${ }^{2}$ Biotechnology/Food Technology Department, Durban University Of Technology, Durban South Africa \\ *Corresponding author: obilanaa@cput.ac.za
}

Received April 11, 2014; Revised April 26, 2014; Accepted May 27, 2014

\begin{abstract}
The objective of this experiment was to assess the effects of malting, extrusion and a combination of both processes on the physical and functional properties of the pearl millet flour (PMF) and pearl millet based instant beverage powder (PMIBP) produced. PMF and PMIBP were prepared by malting, extrusion and a combination of malting and extrusion cooking from two different varieties of pearl millet (Agrigreen (AgG) and Babala (Ba)). Malted pearl millets were produced by germinating at $30^{\circ} \mathrm{C}$ and $98 \% \mathrm{RH}$ for $36 \mathrm{~h}$, kilned at $50^{\circ} \mathrm{C}$ for $48 \mathrm{~h}$ then cooled to room temperature, ground and stored in a chiller at $5^{\circ} \mathrm{C}$ until used. Extrusion was carried out using a corotating twin-screw extruder under different parameters to obtain the pearl millet instant beverage powder. The different processes significantly ( $\mathrm{p} \leq 0.05$ ) affected the colour (L, a \& b) of AgG and Ba. Malting and extrusion significantly $(\mathrm{p} \leq 0.05)$ reduced the peak viscosity of the starches from the raw pearl millet of both varieties of pearl millet as measured by rapid visco analyser (RVA). This can be considered as advantageous with respect to producing an instant beverage powder with a high nutrient content.
\end{abstract}

Keywords: pearl millet, instant, extrusion, malting, beverage

Cite This Article: A.O. OBILANA, B. ODHAV, and V.A. JIDEANI, "Functional and Physical Properties of Instant Beverage Powder Made From Two Different Varieties of Pearl Millet." Journal of Food and Nutrition Research, vol. 2, no. 5 (2014): 250-257. doi: 10.12691/jfnr-2-5-7.

\section{Introduction}

Extrusion cooking, particularly in the snack food industry, is a complex process that differs from conventional processing by using high shear rates and high temperatures $\left(>150^{\circ} \mathrm{C}\right)$ for very short periods (seconds) [1]. Wide ranges of thermo-mechanical and thermo-chemical processes are involved during the extrusion cooking process, including shear, Maillard reactions, protein denaturation and hydrolysis. These processes result in the physical, chemical and nutritional modification of food constituents [2]. Extrusion cooking is a versatile, efficient method of converting raw materials intofinished food products [3], with advantages that include energy efficiency, lack of process effluents and versatility with respect to ingredient selection and the shapes and textures of products that can be produced, Extruded product quality can vary considerably depending onthe extruder type, screw configuration, feed moisture and temperature in the barrelsession, screw speed and feed rate [4]. Extrusion cooking can maketraditional products to be more acceptable in the fast changing society [3]. The extrusion cooking process will be employed in this study to produce pearl millet instant beverage powder (PMIBP).
Malting is the germination of cereal grains in moist air under controlled conditions, followed by drying at $50^{\circ} \mathrm{C}$ for a predetermined length of time. The primary objective of the germination step is to promote the development of hydrolytic enzymes that are not present in the ungerminated grain, whilst the drying is to arrest the enzymatic activity as well as for flavour development.

The objective of this experiment was to assess the effects of malting, extrusion and a combination of both processes on some of the functional and physical properties of the pearl millet flours produced.

\section{Materials and Methods}

\subsection{Source of Pearl Millet Grains, Chemicals and Equipment}

Two different varieties of pearl millet (Pennisetum glaucum) Babala and hybrid Babala (Agrigreen) were obtained from Agricol Pty. Ltd. Cape Town, South Africa. All chemical reagents were purchased from SigmaAldrich South Africa. All equipment used were from the Department of Food Technology, Cape Peninsula University of Technology, Bellville South Africa and CSIR, Pretoria South Africa. 


\subsection{Cleaningand Milling of the Pearlmillet}

The raw pearl millet (RPM) grains were placed in a tray and the chaff and damaged grains as well as stones/pebbles together with all other extraneous matter were removed by hand and discarded.Milling of grains and extrudates to produce flour, was carried out using a hammer mill (CSIR, Sprechert\&Schuh, Germany), with a $2 \mathrm{~mm}$ mesh.

\subsection{Malting Procedure for the Pearl Millet Varieties}

Cleaned grains (10 kg) from each variety, were steeped in $3 \% \mathrm{NaOH}(3 \mathrm{~L})$ at room temperature $\left(23-26^{\circ} \mathrm{C}\right)$ for $3 \mathrm{~h}$ in $10 \mathrm{~L}$ container. After steeping, the grains were drainedthrough cheesecloth and washed thoroughly under running tap water, then allowed to drain off excess water. Three tier plastic vegetable racks $(300 \mathrm{~mm} \times 400 \mathrm{~mm} \times 100$ $\mathrm{mm}$ ) (Plastics Warehouse, Cape Town) were lined with cheesecloth and the grains were then spread on them. The plastic vegetable racks were then put in to a proofing oven (CPUT, MacAdams, Cape Town) at $98 \% \mathrm{RH}$ and $30^{\circ} \mathrm{C}$ for $36 \mathrm{~h}$.

Every $12 \mathrm{~h}$ during the germination period, the millet was rinsed under running tap water, then drained properly and returned to the proofing oven. After $36 \mathrm{~h}$, the grains were spread out evenly on a stainless steel tray, which was then placed in a forced air cabinet drier (CPUT, Geiger Klotzbucher, Cape Town) at $50^{\circ} \mathrm{C}$ for $48 \mathrm{~h}$. After drying, the malted pearl millet (MPM) grains were vacuumpacked and stored in the cold room at $5^{\circ} \mathrm{C}$ until analysed.

\subsection{Extrusion and Combination Processing of the Pearl Millet}

For the extrusion, flours of the raw pearl millet (RPM), malted pearl millet (MPM) and a mixture (50:50) of RPM and MPM from the two varieties were placed into the hopper of a counter rotating extrusion cooker (CSIR, Werner \& Thleiderer, Germany). The processing parameters of the extruder during the extrusion process differed for each of the different samples and were as shown in Table 1. Screw speed (400rpm), feed rate (14.2 $\mathrm{kg} / \mathrm{h})$, heater 1 temperature $\left(100^{\circ} \mathrm{C}\right)$ were kept constant for all extruded flour samples, whilst $\mathrm{H}_{2} \mathrm{O}$ dose rate (8.7 and $12.5 \mathrm{l} / \mathrm{h})$ and heater 2 temperature $\left(110\right.$ to $\left.140^{\circ} \mathrm{C}\right)$ were adjusted to accommodate the level of starch present in each flour sample (the malted PMF have less starch). After extrusion, the extrudates were collected and dried in a forced air cabinet drier (CSIR, Geiger Klotzbucher, Pretoria) at $50^{\circ} \mathrm{C}$ for 24 hours and then milled. Products derived from the extrusion process included: extruded pearl millet (ExPM), extruded malted pearl millet (EMPM) and extruded RPM and MPM50:50 mix (ERPMMPM). Expansion ratio (ER) for the extruded samples (ExPM, EMPM and ERPMMPM) was determined, and all samples (RPM, ExPM, EMPM and ERPMMPM) were analysed for water absorption index (WAI), water solubility index (WSI), colour, colour difference and pasting properties.

Table 1. Processing Parameters of Extruder for The Different Samples

\begin{tabular}{|c|c|c|c|c|c|c|}
\hline & \multicolumn{3}{|c|}{ BABALA } & \multicolumn{3}{|c|}{ AGRIGREEN } \\
\hline & Raw & Malted & Mix & Raw & Malted & Mix \\
\hline Screw speed (rpm) & 400 & 400 & 400 & 400 & 400 & 400 \\
\hline Feed Rate (kg/h) & 14.2 & 14.2 & 14.2 & 14.2 & 14.2 & 14.2 \\
\hline $\mathrm{H}_{2} \mathrm{O}$ dose rate $(\mathrm{l} / \mathrm{hr})$ & 12.5 & 8.7 & 8.7 & 12.5 & 8.7 & 8.7 \\
\hline Heater $1\left({ }^{\circ} \mathrm{C}\right)$ & 100 & 100 & 100 & 100 & 100 & 100 \\
\hline Heater $2\left({ }^{\circ} \mathrm{C}\right)$ & 140 & 120 & 130 & 140 & 110 & 130 \\
\hline Barrel Temp $\left({ }^{\circ} \mathrm{C}\right)$ & 83 & 85.5 & 84.6 & 84.3 & 84.3 & 85 \\
\hline Product Temp $\left({ }^{\circ} \mathrm{C}\right)$ & 91 & 93 & 91 & 94 & 90 & 91 \\
\hline Torque (N) & 20 & 28 & 34 & 20 & 20 & 20 \\
\hline Die Size (mm) & 5 & 5 & 5 & 5 & 5 & 5 \\
\hline
\end{tabular}

2.5. Water Absorption Index, Water Solubility Index, and Expansion Ratio of the Pearl Millet Flour and Pearl Millet Instant Beverage Powder

The water absorption index (WAI) of the RPM, ExPM, MPM, EMPM and ERPMMPM were determined by the method of [5]. A sample (2.5 g) was suspended in $30 \mathrm{ml}$ distilled water at $30^{\circ} \mathrm{C}$ in a previously weighed $50 \mathrm{ml}$ centrifuge tube. This mixture was stirred intermittently over a $30 \mathrm{~min}$ period and then centrifuged (Thermo Electron, France) at $3000 \mathrm{~g}$ for $10 \mathrm{~min}$. After pouring the supernatant into a tarred evaporating dish, the gel was weighed and the WAI defined as the grams of gel per gram of solids was calculated as follows:

$$
\text { WAI }(\% \text { d.b. })=\frac{\text { Weight of Sediment }}{\text { Weight of dry solids }} \times 100
$$

Flour WSI, defined, as the water-soluble fraction in the sample extract, was determined from the amount of dried solids recovered by evaporating the supernatant from the flour water absorption test [5]. The WSI was calculated as follows:

$$
\begin{aligned}
& \text { WSI }(\% \text { d.b. }) \\
= & \frac{\text { Weight of solids dissolved in supernatant }}{\text { Weight of dry solids }} \times 100
\end{aligned}
$$

The expansion ratio for the extrudates was determined according to the method of [6]. This was determined by measuring the diameter ( $\mathrm{mm}$ ) of the extrudates using vernier callipers and then dividing these readings by the diameter of the extruder nozzle. Ten readings were determined for each sample and their mean recorded. The ER was calculated as:

$$
\text { Expansion Ratio }(E R)=\frac{\text { Extrudate diameter }}{\text { Die diameter }}
$$


The colour of the RPM, ExPM, MPM, EMPM and ERPMMPM were analysed using a Konica Minolta Spectrophotometer (model CM 5, Konica Minolta Sensing Inc., Japan). An adequate amount of each sample was placed in the sample holder (diameter $30 \mathrm{~mm}$ ) to cover the base and allow for reflectance measurement. Measurements for each individual sample were performed in triplicate by rotating the dish to three different positions (one reading $=$ average of three readings per rotated position). The colour was recorded in terms of Hunter L, a and b. L (lightness of the product from 0 for black to 100 for perfect white), a (chromaticity coordinate $+a=$ red and $-\mathrm{a}=$ green), $\mathrm{b}$ (chromaticity coordinate $+\mathrm{b}=$ yellow and $\mathrm{b}=$ blue) [7].

The colour difference, a measure of the difference in colour between the different samples and control (RPM from both varieties) was calculated from the data collected using the following equation:

$$
\text { Colour difference }(\Delta E)=\sqrt{\Delta L^{2}+\Delta a^{2}+\Delta b^{2}}
$$

Where:

$$
\begin{aligned}
& \mathrm{L}=\text { lightness } \\
& \mathrm{a}=\text { redness/greenness } \\
& \mathrm{b}=\text { yellowness/ blueness }
\end{aligned}
$$

\subsection{Pasting Properties of the Pearl Millet Flours (PMF) and Pearl Millet Instant Beverage Powder (PMIBP)}

The pasting properties of the RPM, ExPM, MPM, EMPM and ERPMMPM were assessed according to the method of [8], using a Rapid Visco Analyser (US, RVA4500, Perten Instruments Australia) with some modifications.Approximately $4 \mathrm{~g}$ of extrudates flour was added to $25 \mathrm{ml}$ of distilled water. The heating and cooling cycles wereprogrammed in the following manner. Each sample washeld at $50^{\circ} \mathrm{C}$ for $1 \mathrm{~min}$, heated to $95^{\circ} \mathrm{C}$ in 3.42 min, heldat $95^{\circ} \mathrm{C}$ for $2.5 \mathrm{~min}$, cooled to $50^{\circ} \mathrm{C}$ in $3.80 \mathrm{~min}$ and maintained at this temperature for $2 \mathrm{~min}$. The total time for analysis was $13 \mathrm{~min}$, during whichtime the following parameters were measured and recorded by the Thermocline software: peak viscosity (PV), holding strength (HS), breakdown viscosity (BV), final viscosity $(\mathrm{FV})$, setback viscosity (SV) and peak time (PT).

\subsection{Data Analyses}

All data were collected in triplicate. The data were subjected to a multivariate analysis to establish whether there were mean differences between treatments. The Duncan multiple range test was used to separate means where differences existed. Categorical Principal Component Analysis (CATPCA) with optimal scaling was used to explore the joint relationships among the observed proximate, physical and functional characteristics and the pearl millet. CATPCA analysis was divided into three parts: quantifications for the categories of the variable scores for the objects (with mean of zero and unit variance) and component loading for the transformed variables. All data analyses were carried out using IBM SPSS Statistics version 21, 2012.

\section{Results and Discussion}

\subsection{Effect of Malting, Extrusion and a Combination Thereof on Water Absorption Index, Water Solubility Index, Expansion Ratio and Colour of the Pearl Millet Flour and Pearl Millet Instant Beverage Powder}

The effect of extrusion, malting and a combination of both processes on the water absorption index (WAI), water solubility index (WSI) and expansion ratio (ER) of RPM, ExPM, MPM, EMPM and ERPMMPM from AgG is summarised in Table 2. The WAI, WSI and ER were significantly $(\mathrm{p} \leq 0.05)$ increased by extrusion and a combination of extrusion and malting. Malting, however, significantly $(\mathrm{p} \leq 0.05)$ increased WSI $(12.2 \%)$ but had no effect on WAI (319.6\%).

Table 2. Water Absorption Index, Water Solubility Index and Expansion Ratio of Pearl Millet (Agrigreen) as Affected byMalting, Extrusion and a Combination Thereof ${ }^{1,2}$

\begin{tabular}{cccc}
\hline $\begin{array}{c}\text { Pearl millet } \\
\text { treatment }\end{array}$ & WAI (\% d.b.) & WSI (\% d.b.) & ER \\
\hline RPM & $284.54 \pm 4.9^{\mathrm{a}}$ & $5.14 \pm 0.7^{\mathrm{a}}$ & ---- \\
ExPM & $650.92 \pm 90.2^{\mathrm{b}}$ & $8.29 \pm 0.8^{\mathrm{a}}$ & $9.01 \pm 0.4^{\mathrm{a}}$ \\
MPM & $319.57 \pm 43.3^{\mathrm{a}}$ & $12.16 \pm 4.6^{\mathrm{b}}$ & ---- \\
EMPM & $414.67 \pm 103.9^{\mathrm{c}}$ & $12.94 \pm 3.9^{\mathrm{b}}$ & $5.71 \pm 0.4^{\mathrm{b}}$ \\
ERPMMPM & $534.30 \pm 82.4^{\mathrm{c}}$ & $13.33 \pm 0.4^{\mathrm{b}}$ & $6.59 \pm 0.4^{\mathrm{c}}$ \\
\hline OTE & $440.80 \pm 154.0$ & $10.37 \pm 4.0$ & $7.11 \pm 1.5$ \\
\hline
\end{tabular}

${ }^{1}$ Values are mean \pm standard deviation. Different superscripts in columns differ significantly $(\mathrm{p} \leq 0.05)$

${ }^{2} \mathrm{RPM}=$ Raw pearl millet; $\mathrm{RE}=$ Extruded pearl millet; $\mathrm{MPM}=$ Malted pearl millet; EMPM = Extruded malted

pearl millet, ERPMMPM = Extruded raw pearl millet-malted pearl millet mix, OTE = Overall treatment effect,

WAI = Water Absorption Index, WSI = Water Solubility Index, ER = Expansion Ratio.

The ER of all the extrusion treatments differed significantly $(p \leq 0.05)$ from each other. Summarised in Table 3 are the effects of extrusion, malting and a combination of both processes on the WAI, WSI and ER of RPM, ExPM, MPM, EMPM and ERPMMPM made from Ba. Similar to trends of the effects of malting, extrusion and a combination of both processes on AgG were observed for $\mathrm{Ba}$, with WAI and WSI being significantly $(\mathrm{p} \leq 0.05)$ increased by both extrusion and $\mathrm{a}$ combination of malting and extrusion. However, malting, as was the case with AgG had no effect on WAI and WSI of Ba. A significant difference was observed amongst all processing methods for ER.

Table 3. Water Absorption Index, Water Solubility Index and Expansion Ratio of Pearl Millet (Babala) as Affected by Malting, Extrusion and a Combination Thereof ${ }^{1,2}$

\begin{tabular}{cccc}
\hline $\begin{array}{c}\text { Pearl millet } \\
\text { treatment }\end{array}$ & WAI (\% d.b.) & WSI (\% d.b.) & ER \\
\hline RPM & $259.16 \pm 8.7^{\mathrm{a}}$ & $6.78 \pm 0.1^{\mathrm{a}}$ & \\
ExPM & $510.10 \pm 2.7^{\mathrm{b}}$ & $11.67 \pm 0.5^{\mathrm{b}}$ & $9.83 \pm 0.3^{\mathrm{a}}$ \\
MPM & $288.76 \pm 10.3^{\mathrm{a}}$ & $13.32 \pm 0.3^{\mathrm{c}}$ & \\
EMPM & $395.85 \pm 24.7^{\mathrm{c}}$ & $16.16 \pm 1.7^{\mathrm{d}}$ & $6.97 \pm 1.7^{\mathrm{b}}$ \\
ERPMMPM & $526.44 \pm 65.2^{\mathrm{b}}$ & $10.16 \pm 2.9^{\mathrm{b}}$ & $8.88 \pm 0.6^{\mathrm{a}}$ \\
\hline OTE & $396.06 \pm 116.8$ & $11.62 \pm 3.5$ & $8.56 \pm 1.6$ \\
\hline
\end{tabular}

${ }^{1}$ Values are mean \pm standard deviation. Different superscripts in columns differ significantly $(\mathrm{p} \leq 0.05)$

${ }^{2} \mathrm{RPM}=$ Raw pearl millet; RE = Extruded pearl millet; $\mathrm{MPM}=$ Malted pearl millet; EMPM = Extruded

malted pearl millet, ERPMMPM = Extruded raw pearl millet-malted pearl millet mix, OTE = Overall

treatment effect, WAI $=$ Water Absorption Index, WSI $=$ Water Solubility Index, ER = Expansion Ratio. 
For both AgG and Ba (Table 2 and Table 3), WAI was significantly $(\mathrm{p} \leq 0.05)$ affected by extrusion, whilst WSI was significantly $(\mathrm{p} \leq 0.05)$ affected by malting. Malting can also be seen to have a significant $(p \leq 0.05)$ effect on ER of the extrudates. The significant $(\mathrm{p} \leq 0.05)$ increase in WAI and WSI is in agreement with results reported by [9] and [10] of increased WAI in extruded samples in their studies. The differences in the results of their work and those obtained in this work, could be attributed to several factors important in developing the characteristics of the extruded product, including the type of extruder, feed moisture, feed rate, barrel temperature, screw speed, screw profile and die size [11]. The changes in the functional and physical properties observed in this study are in agreement with the observation that during extrusion processing, food materials are generally subjected to a combination of high temperature, high pressure and high shear, resulting in a variety of reactions with corresponding changes in the functional properties of the extruded material [11]. The increase in water absorption in this work is one of the many changes in physical and functional properties that occur because of extrusion, and this is in agreement with [12], who also observed a general increase in water absorption of sorghum extrudates.

High WAI for extruded pearl millet in this work are further corroborated by [13], who concluded that gelatinized starch readily absorbs water to form paste that had higher final viscosity at room temperature than native starch. The reductions in ER during extrusion can be explained by the fact that protein acts as 'filler' in cereal extrudates and is dispersed in the continuous phase of the extrusion melt, modifying the flow behaviour and characteristics of the cooled extrudates [14]. Proteinaceous materials hydrate in the mixing stage of the process and become soft viscoelastic doughs during the formation of the extrusion melt. The shearing forces generated in the extruder cause breakage of the protein into small particles of roughly cylindrical and globular shapes. At protein levels of 5-15\%, the extensibility of the starch polymer foam during its expansion at the die exitis reduced, reducing the degree of expansion. This conclusion is also in agreement with the report that extrudates show a reduction in expansion with increasing protein content [11]. At higher levels of protein, severely torn regions in the cell walls of the extrudate are noted, indicating a loss of elasticity in the extrusion melt [11]. The low ER observed in EMPM (which had a lower fat content than un-malted pearl millets for both varieties) is in agreement with [3] who reported low puff ratios in extruded high fat content cereal grain-legume mixture. This is also in agreement with the report that the expanded volume of cereal flour decreases with increasing amounts of protein and lipid but increases with starch content [15]. The lower expansion ratio in the mixtures of soybean and groundnut in their work may be attributed to lower protein content of blends of cowpea compared to those of soybean and groundnut except for $100 \%$ millet, which recorded the highest expansion ratio. The fat content in the malted grain in this experiment was found to be lower than that in the un-malted grain. Going by the observations made by [3] and [15], the ER for the malted grain should be higher than the un-malted grain. But this was not the case in this study, because the malting process does not only breakdown and reduce fat content, but also the starch present in the grain is converted to dextrins simultaneously. Dextrins do not behave like starch under cooking conditions; they do not hold as much water as starch molecules, but dissolve easier in water and under less stringent conditions than starch molecules. Hence, the differing effects of malting and extrusion on WAI, WSI and ER (Table 2 and Table 3). High temperature extrusion enables starch to becomemore fully cooked and thus better able to expand.

\subsection{Effect of Malting, Extrusion and a Combination Thereof on the Colour of the Instant Beverage Powder}

Table 4 and Table 5 summarises the effect of malting, extrusion and a combination of these two processes on the colour of RPM, ExPM, MPM, EMPM and ERPMMPM from both AgG and Ba. For the AgG samples (Table 4), there was a significant $(\mathrm{p} \leq 0.05)$ difference in the lightness $(\mathrm{L})$ of the product from $72.89 \pm 0.0$ for EMPM to $86.22 \pm 0.0$ for MPM. The Ba samples (Table 5) exhibited similar trends to the AgGsamples Thelightness of the products ranged from $71.60 \pm 0.06$ for ERPMMPM to $86.58 \pm 0.01$ for RPM. All processing methods caused a decrease in lightness $(\mathrm{L})$ values, with the extruded (ExPM, EMPM and ERPMMPM) products been the lowest. The redness $( \pm \mathrm{a})$ of the products ranged from $-0.51 \pm 0.0$ for RPM to $3.03 \pm 0.0$ for ERPMMPM, an indication of processing methods having a significant effect. The yellowness $( \pm b)$ of the products ranged from $15.23 \pm 0.0$ to $22.53 \pm 0.0$ for AgG RPM and AgG EMPM, respectively and $16.41 \pm 0.0$ to $33.34 \pm 0.0$ for Ba MPM to Ba ExPM, respectively.

Malting caused a significant $(p \leq 0.05)$ decrease in yellowness whilst extrusion and a combination of both processes increased it significantly ( $\mathrm{p} \leq 0.05$ ). According to [16], colour is an important characteristic of extruded foods, which can give information about theextent of browning reactions such as caramelisation, Maillard reaction, degree of cooking and pigment degradation during the extrusion process. Hence the colour differences between extruded and non-extruded products, could be due to the shear forces generated during extrusion which accelerated the chemical reactions between amino acids and reducing sugars (Maillards reaction) that take place during extrusion and to the different cooking temperature, rolling speed, flow rate, water dosing rate and feeding speed conditions during extrusion $[17,18]$.

The estimated colour difference for RPM, ExPM, MPM, EMPM and ERPMMPM from both AgG and Ba indicates the change in colour from the RPM flour. All the processes (malting, extrusion and a combination of both) had a significant $(\mathrm{p} \leq 0.05)$ effect on colour (Table 4 and Table 5). The effect of extrusion on the colour change in RPM, during processing to ExPM, MPM, EMPM and ERPMMPM from both varieties of pearl millet was significantly higher than the effect of malting. This difference could be attributed to the high temperatures used during extrusion in conjunction with the presence of sugars (malted samples) and amino acids leading to Maillard browning of the final products. The lack of high temperatures during malting would mean less browning during this process. 
Table 4. Colour Of Pearl Millet (Agrigreen) as Affected by Malting, Extrusion and a Combination of both Processing Methods ${ }^{1,2}$

\begin{tabular}{|c|c|c|c|c|}
\hline \multirow[b]{2}{*}{ Pearl millet treatment } & \multicolumn{4}{|c|}{ Colour } \\
\hline & $\mathrm{L}$ & $\mathrm{a}$ & $\mathrm{b}$ & Colour Difference $(\Delta \mathrm{E})$ \\
\hline RPM & $81.38 \pm 0.01^{\mathrm{a}}$ & $0.95 \pm 0.01^{\mathrm{a}}$ & $15.23 \pm 0.02^{\mathrm{a}}$ & \\
\hline ExPM & $75.77 \pm 0.01^{\mathrm{b}}$ & $1.15 \pm 0.00^{\mathrm{b}}$ & $20.95 \pm 0.02^{b}$ & $13.48 \pm 0.00^{\mathrm{a}}$ \\
\hline MPM & $86.22 \pm 0.06^{\mathrm{c}}$ & $1.26 \pm 0.01^{\mathrm{c}}$ & $15.27 \pm 0.01^{\mathrm{a}}$ & $7.71 \pm 0.04^{\mathrm{b}}$ \\
\hline EMPM & $72.89 \pm 0.08^{d}$ & $2.50 \pm 0.01^{\mathrm{d}}$ & $22.53 \pm 0.02^{\mathrm{c}}$ & $15.36 \pm 0.04^{\mathrm{c}}$ \\
\hline ERPMMPM & $73.92 \pm 0.01^{\mathrm{e}}$ & $2.32 \pm 0.00^{\mathrm{e}}$ & $22.34 \pm 0.04^{\mathrm{d}}$ & $15.45 \pm 0.06^{\mathrm{d}}$ \\
\hline OTE & $78.04 \pm 5.21$ & $1.64 \pm 0.66$ & $19.26 \pm 3.44$ & $10.40 \pm 6.12$ \\
\hline
\end{tabular}

${ }^{1}$ Values are mean \pm standard deviation. Different superscripts in columns differ significantly $(\mathrm{p} \leq 0.05)$

${ }^{2} \mathrm{RPM}=$ Raw pearl millet; RE = Extruded pearl millet; MPM = Malted pearl millet; EMPM = Extruded malted

pearl millet, ERPMMPM = Extruded raw pearl millet-malted pearl millet mix, OTE = Overall treatment effect.

Table 5. Colour of Pearl Millet (Babala) as Affected by Malting, Extrusion and a Combinationof both Processing Method ${ }^{1,2}$

\begin{tabular}{|c|c|c|c|c|}
\hline \multirow[b]{2}{*}{ Pearl millet treatment } & \multicolumn{4}{|c|}{ Colour } \\
\hline & $\mathrm{L}$ & a & b & Colour Difference $(\Delta \mathrm{E})$ \\
\hline RPM & $86.58 \pm 0.01^{\mathrm{a}}$ & $-0.51 \pm 0.01^{\mathrm{a}}$ & $23.94 \pm 0.01^{\mathrm{a}}$ & \\
\hline ExPM & $77.13 \pm 0.02^{\mathrm{b}}$ & $1.56 \pm 0.01^{\mathrm{b}}$ & $33.34 \pm 0.02^{b}$ & $8.02 \pm 0.00^{\mathrm{a}}$ \\
\hline MPM & $86.70 \pm 0.01^{\mathrm{c}}$ & $1.15 \pm 0.01^{\mathrm{c}}$ & $16.41 \pm 0.04^{\mathrm{c}}$ & $4.84 \pm 0.06^{\mathrm{b}}$ \\
\hline EMPM & $71.76 \pm 0.03^{\mathrm{d}}$ & $2.09 \pm 0.01^{\mathrm{d}}$ & $27.03 \pm 0.02^{\mathrm{d}}$ & $11.30 \pm 0.06^{\mathrm{c}}$ \\
\hline ERPMMPM & $71.60 \pm 0.06^{\mathrm{e}}$ & $3.03 \pm 0.00^{\mathrm{e}}$ & $25.27 \pm 0.10^{\mathrm{e}}$ & $10.39 \pm 0.01^{\mathrm{d}}$ \\
\hline OTE & $78.75 \pm 6.9$ & $1.46 \pm 1.2$ & $25.20 \pm 5.6$ & $6.91 \pm 4.3$ \\
\hline
\end{tabular}

${ }^{1}$ Values are mean \pm standard deviation. Different superscripts in columns differ significantly (p $\leq 0.05$ )

${ }^{2} \mathrm{RPM}=$ Raw pearl millet; RE = Extruded pearl millet; MPM = Malted pearl millet; EMPM = Extruded malted pearl

millet, ERPMMPM = Extruded raw pearl millet-malted pearl millet mix, OTE = Overall treatment effect.

The colour difference is also a measure of the acceptability of the RPM, ExPM, MPM, EMPM and ERPMMPM by the consumer. If $\Delta \mathrm{E} \geq 1$, it is generally regarded that the consumer will perceive a colour difference between the product and control [16]. The colour difference was greater than 1 for all samples, an indication of the perception of a colour difference by the consumer in all the pearl millet samples.

The pasting properties for AgG and Ba are summarised in Table 6 and Table 7, respectively. For AgG (Table 6), peak viscosity ranged from $42.33 \pm 2.08$ for malted pearl millet (MPM) to $719.33 \pm 11.59$ RVUfor raw pearl millet (RPM). The holding strength ranged from $22.00 \pm 0.00$ for MPM to $657.67 \pm 11.59$ RVU for (RPM). The breakdown viscosity ranged from $19.33 \pm 1.15$ for extruded pearl millet malt (EMPM) to $61.67 \pm 2.89 \mathrm{RVU}$ for (RPM). The final viscosity ranged from $36.33 \pm 1.15$ for (MPM) to 3.3. Effect of Malting, Extrusion and a Combination Thereof on the Pasting Properties of the Pearl Millet (AgriGreen and Babala) Flour $1826.00 \pm 45.18 \mathrm{RVU}$ for (RPM). The setback viscosity was between $14.33 \pm 1.15$ (MPM) and $1168 \pm 33.61$ RVU (RPM) and the peak time ranged from $3.66 \pm 0.46$ minutes for the extruded pearl millet (ExPM) to $5.38 \pm 0.04$ minutes for the raw pearl millet (RPM).

Table 6. Pasting Properties of Pearl Millet (Agrigreen) as Affected by Malting, Extrusion and a Combination of both Processing Methods ${ }^{1,2}$

\begin{tabular}{|c|c|c|c|c|c|c|}
\hline Pearl millet Treatment & $\begin{array}{c}\text { Peak Viscosity } \\
\text { (RVU) }\end{array}$ & $\begin{array}{c}\text { Trough / Holding } \\
\text { strength (RVU) }\end{array}$ & Breakdown (RVU) & Final Viscosity (RVU) & Setback (RVU) & Peak Time (min) \\
\hline RPM & $719.33 \pm 11.59^{\mathrm{a}}$ & $657.67 \pm 11.59^{\mathrm{a}}$ & $61.67 \pm 2.89^{a}$ & $1826.00 \pm 45.18^{a}$ & $1168.33 \pm 33.61^{a}$ & $5.38 \pm 0.04^{\mathrm{a}}$ \\
\hline ExPM & $97.67 \pm 6.66^{b}$ & $63.00 \pm 1.00^{\mathrm{b}}$ & $34.67 \pm 5.69^{b}$ & $93.00 \pm 5.00^{b}$ & $30.00 \pm 5.57^{b}$ & $3.66 \pm 0.46^{\mathrm{b}}$ \\
\hline MPM & $42.33 \pm 2.08^{c}$ & $22.00 \pm 0.00^{c}$ & $20.33 \pm 2.08^{c}$ & $36.33 \pm 1.15^{\mathrm{c}}$ & $14.33 \pm 1.15^{\mathrm{b}}$ & $3.67 \pm 0.00^{b}$ \\
\hline EMPM & $73.33 \pm 1.53^{d}$ & $54.00 \pm 1.00^{\mathrm{d}}$ & $19.33 \pm 1.15^{c}$ & $88.00 \pm 2.00^{b}$ & $34.00 \pm 2.65^{b}$ & $3.96 \pm 0.08^{\mathrm{b}}$ \\
\hline ERPMMPM & $67.67 \pm 4.04^{\mathrm{d}}$ & $48.00 \pm 1.00^{\mathrm{d}}$ & $19.67 \pm 3.51^{c}$ & $86.00 \pm 8.19^{b}$ & $38.00 \pm 7.55^{b}$ & $4.05 \pm 0.17^{\mathrm{b}}$ \\
\hline OTE & 200.06 & 168.93 & 31.13 & 425.87 & 256.93 & 4.14 \\
\hline
\end{tabular}

${ }^{1}$ Values are mean \pm standard deviation. Different superscripts in columns differ significantly $(\mathrm{p} \leq 0.05)$
${ }^{2} \mathrm{RPM}=$ Raw pearl millet; RE = Extruded pearl millet; $\mathrm{MPM}=$ Malted pearl millet; EMPM = Extruded malted pearl millet, ERPMMPM = Extruded
raw pearl millet-malted pearl millet mix, OTE = Overall treatment effect.

Table 7. Pasting Properties of Pearl Millet (Babala) as Affected by Malting, Extrusion and a Combination of both Processing Methods ${ }^{1,2}$

\begin{tabular}{|c|c|c|c|c|c|c|}
\hline $\begin{array}{c}\text { Pearl millet } \\
\text { Treatment }\end{array}$ & $\begin{array}{c}\text { Peak Viscosity } \\
\text { (RVU) }\end{array}$ & $\begin{array}{c}\text { Trough / Holding } \\
\text { strength (RVU) }\end{array}$ & Breakdown (RVU) & $\begin{array}{c}\text { Final Viscosity } \\
\text { (RVU) }\end{array}$ & Setback (RVU) & Peak Time (min) \\
\hline $\mathrm{RPM}^{2}$ & $932.67 \pm 23.59^{\mathrm{a} 1}$ & $843.67 \pm 11.37^{\mathrm{a}}$ & $89.00 \pm 12.53^{\mathrm{a}}$ & $2009.33 \pm 49.80^{\mathrm{a}}$ & $1165.67 \pm 46.82^{\mathrm{a}}$ & $5.55 \pm 0.04^{\mathrm{a}}$ \\
\hline ExPM & $78.33 \pm 3.06^{\mathrm{b}}$ & $62.67 \pm 1.52^{\mathrm{b}}$ & $15.67 \pm 1.52^{\mathrm{b}}$ & $109.33 \pm 2.52^{b}$ & $46.67 \pm 2.08^{\mathrm{b}}$ & $4.47 \pm 0.18^{\mathrm{b}}$ \\
\hline MPM & $29.75 \pm 7.54^{\mathrm{c}}$ & $12.50 \pm 9.81^{\mathrm{c}}$ & $17.25 \pm 2.36^{\mathrm{b}}$ & $34.75 \pm 22.20^{c}$ & $22.25 \pm 12.61^{\mathrm{b}}$ & $3.72 \pm 0.06^{\mathrm{c}}$ \\
\hline EMPM & $46.33 \pm 12.70^{\mathrm{d}}$ & $30.33 \pm 6.65^{d}$ & $16.00 \pm 6.08^{b}$ & $64.67 \pm 2.52^{c}$ & $34.33 \pm 5.03^{b}$ & $3.73 \pm 0.17^{c}$ \\
\hline ERPMMPM & $61.00 \pm 1.41^{\mathrm{b}}$ & $39.50 \pm 2.12^{d}$ & $21.50 \pm 3.53^{b}$ & $73.00 \pm 4.24^{\mathrm{b}}$ & $33.50 \pm 2.12^{b}$ & $4.10 \pm 0.04^{d}$ \\
\hline OTE & 227.53 & 195.93 & 31.60 & 455.67 & 259.73 & 4.29 \\
\hline
\end{tabular}

\footnotetext{
${ }^{1}$ Values are mean \pm standard deviation. Different superscripts in columns differ significantly $(\mathrm{p} \leq 0.05)$

${ }^{2} \mathrm{RPM}=$ Raw pearl millet; RE = Extruded pearl millet; MPM = Malted pearl millet; EMPM = Extruded malted pearl millet, ERPMMPM = Extruded raw pearl millet-malted pearl millet mix, OTE = Overall treatment effect.
} 
In general, the raw PMF of both varieties exhibited the highest (significant $(\mathrm{p} \leq 0.05)$ peak, trough, breakdown, final and setback viscosities; as well as peak time. The malted PMF and malted PMIBP however, exhibited the lowest values for the same parameters. These trends are similar to those observed by [19] in their study of complementary foods made from malted and un-malted millet with soybean and cowpea. They observed that blends, which were based on the whole millet grain, had a higher peak viscosity than blends based on the germinated millet flour, and the paste stability of the blend based on the germinated millet flour was higher than blends based on the whole grain. This reduction in viscosity of the MPM, EMPM and ERPMMPM can be attributed to starch degradation by amylases during the germination process.

The viscosity of a paste depends to a large extent on the degree of gelatinization of the starch granules and the rate of molecular breakdown. In addition to the effect of extrusion, the reduction in viscosity may be attributed to the high level of fat in the millet grains, which consequently decreased the shear effect because of lubrication in the metering zone.Increase in moisture on the other hand, will further lubricate the dough leading to less shearing effect [20]. Low moisture in the feed can possibly increase frictional damage, particularly when the residence time is high due to low screw speed. Viscosity generally depends on solubility and water holding capacity as well as the structure of components in a food system. Viscosity profile can be thought of as a reflection of the granular changes in the starch granule that occur during gelatinization [21].
The decrease in peak and final viscosity of the ExPM, EMPM and ERPMMPM, could be as a result of the dextrinization of starches in the PMF during extrusion, as a result of the high temperature and pressures. This is in agreement with the report that extrusion can induce starch dextrinization resulting in reduction of viscosity in gruels and a concomitant increase in caloric and nutrient density [22,23], as well asdecreased apparent viscosity of extruded instant corn flour when temperature was increased [24]. Viscosity over a heating and cooling cycle have been used to characterise the changes in extruded products in numerous studies [25]. This characteristic is affected by both physical modifications of the granule structure as well as changes to the structures of the starch polymers.Furthermore, the characteristics of the paste viscosity curves were significantly altered by extrusion processing with extrudates showing low values [24,25]. This is in agreement with observations made in this experiment, with RPM for both varieties showing significantly $(\mathrm{p} \leq 0.05)$ higher viscosity values than the samples that were extruded, malted or processed by a combination of both methods. The reduction in viscosity of the extruded samples may be because of dextrinization of the starch molecules in pearl millet, which agrees with observations made by [22,23]. Starchdextrinization during extrusion cooking, however, occurred mostly under processing conditions at very high temperature and low moisture, where shear effects were significant [26] which are similar to the extrusion conditions used during this experiment. This reduction in viscosity could be beneficial for infant feeding[27]or for any person(athletes or patients) requiring a liquid diet with a high nutritional value.

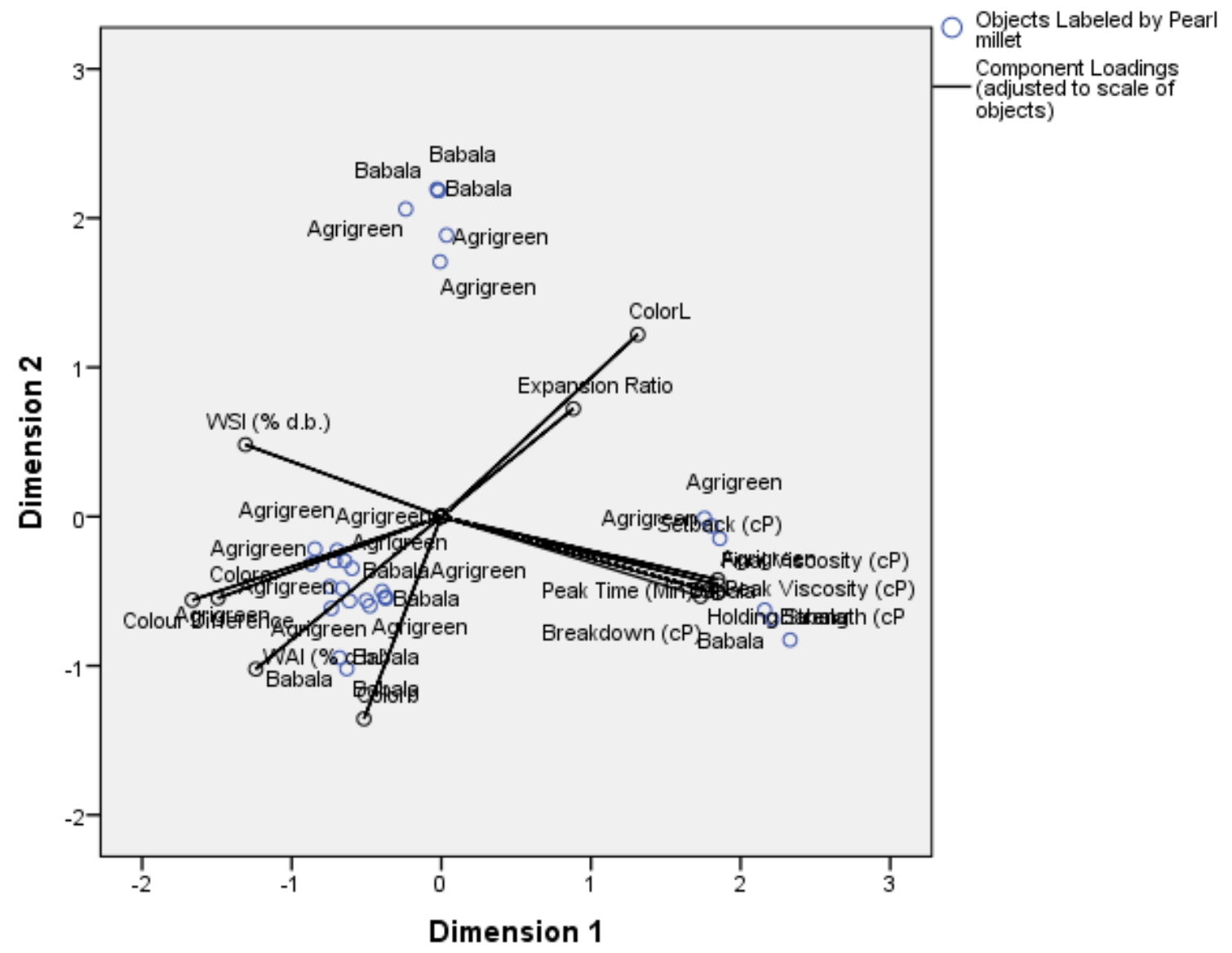

Figure 1. Biplot component loadings (physical and functional properties) and objects labelled by pearl millet variety. Circles indicate positive correlation between enclosed components 


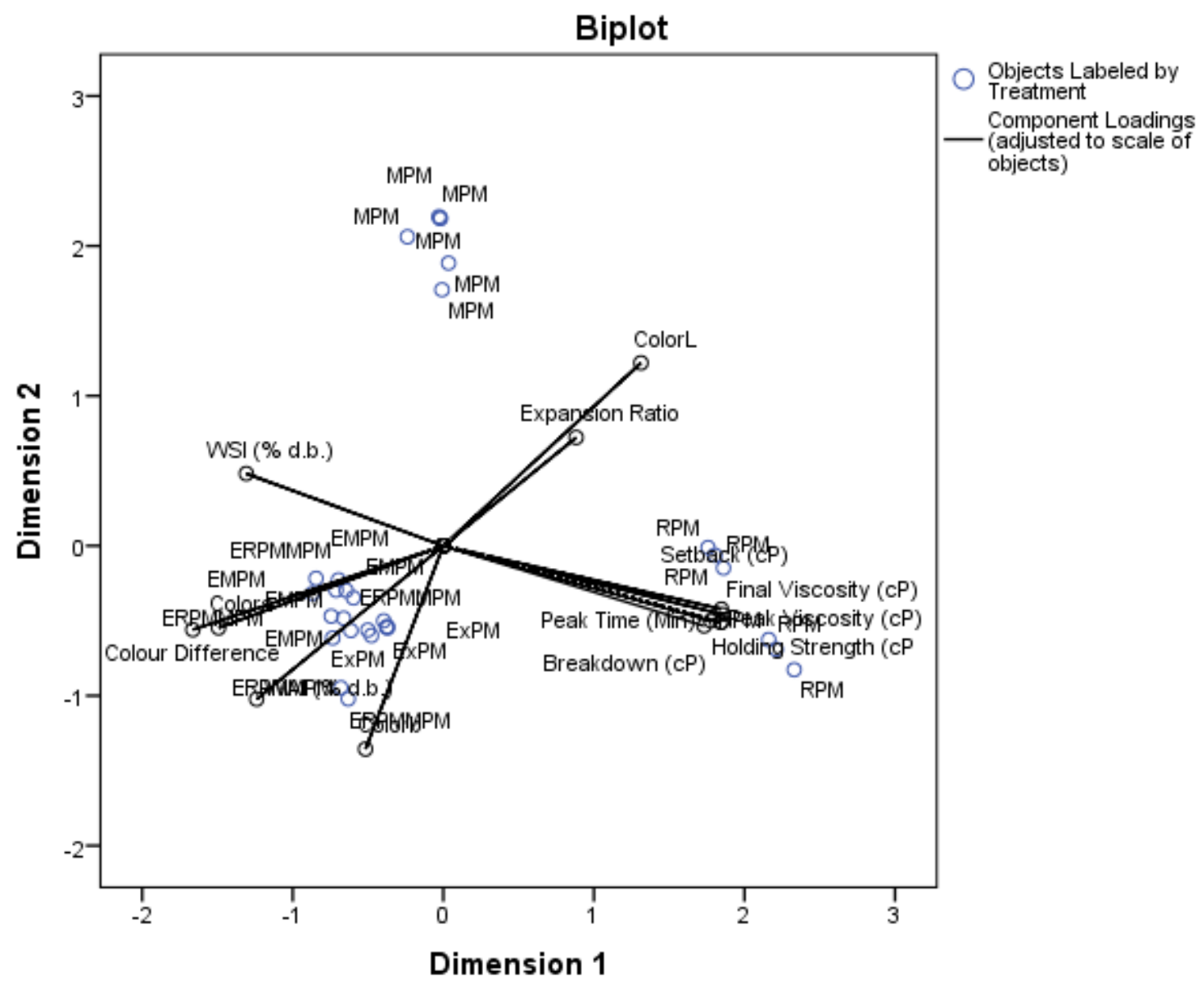

Figure 2. Biplot component loadings (physical and functional properties) and objects labelled by treatment. Circles indicate positive correlation between enclosed components

Figure 1 and Figure 2 details geometrical display that allows a visual interpretation of relationships between categories of pearl millet varieties, treatment and observed variables - physical and functional properties, as well as their relationship to the two dimensions. Component loadings and Pearson correlations ranged from -2 to 3 in the two dimensional solution. The coordinates of the endpoint of each vector are given by the loadings of each variable on the first and second dimensions. The variables closely grouped together in the plot are positively correlated. Vectors making a 90-degree angle are not correlated.

The inherent relationship could be described by two components accounting for $84.2 \%$ variation. The variance accounted for by dimension, 1 is $68.3 \%$ and dimension 2 is $15.9 \%$. Dimension 1 is positively correlated to $\mathrm{Ba}$ and AgG RPM high in PV, FV, HV, SV, BV and PT (Figure 1). The correlation of dimension 1 with AgG and Ba RPM with high viscosity values is expected, as the starch in the RPM is in its native form and gelatinises to form a viscous paste. Processing the pearl millet by malting, extrusion or a combination of both processes on the other hand, resulted in degradation of the starch molecules to dextrins, which are shorter chain polysaccharides than starch. This led to the formation of a thin watery paste on gelatinisation, hence the lower viscosities and negative correlation to dimension 1 . Dimension 2 is positively correlated to MPM of bothvarieties of pearl millet, high in lightness (L) and ER. Hence malting resulted in lighter products. From Figure 2, it is clear that extruded products (ExPM, EMPM and ERPMMPM) were darker in colour compared to the RPM and MPM as they correlated to a high colour difference (Dimension 2).

\section{Conclusions and Recommendations}

Malting and extrusion reduced the viscosity of RPM as indicated by the low viscosities of ExPM, MPM, EMPM and ERPMMPM made from both varieties of pearl millet. This is advantageous with respect to an increase in nutrient density of the resulting beverages prepared from them. This would be suitable for use in the combatting of energy malnutrition observed mostly in young children of low income communities.

Processing of flour from RPM, ExPM, MPM, EMPM and ERPMMPM from both varieties of pearl millet by malting, extrusion and a combination of both processes under the parameters used, had both beneficial and/or no effects on the functional and physical properties. Final viscosity of RPM was higher than that of ExPM, MPM, EMPM and ERPMMPM, an indication of damage to the native starch by the processing methods used. Malting lightened the colour of the RPM with high WSI, ER and lower viscosity. Extrusion produced darker products (especially ExPM) with perceivable colour differences from the RPM.

Manipulating processing parameters such as barrel temperature or screw speed amongst others during extrusion processing; or steeping conditions or germination time during malting, would alter the effects of these processes on the properties determined. If the effects 
are variety dependent (not ascertained in this experiment), development of varieties specifically suited for beverage production will have to be investigated in order to reap maximum benefits from the processes and their advantages.

\section{Acknowledgement}

Durban University of Technology, Biotechnology / Food Technology Department Cape Peninsula University of Technology, Food Technology department. Stellenbosh University, Food Science Department.

\section{References}

[1] Athar, N., et al., Vitamin retention in extruded food products. Journal of Food Composition and Analysis, 2006. 19: p. 379-383.

[2] Linko, P., P. Colonna, and C. Mercier, High temperature, short time extrusion-cooking. Advances in Cereal Science and Technology, 1981. 4: p. 145-235.

[3] Filli, K.B. and I. Nkama, Hydration properties of extruded fura from millet and legumes. British Food Journal, 2007. 109 (1): p. 68-80.

[4] Qing-Bo, D., et al., The effect of extrusion conditions on the physicochemical properties and sensory characteristics of ricebased expanded snacks. Journal of Food Engineering, 2005. 66: p. 284-289.

[5] Anderson, R.A., et al., Roll and extrusion cooking of grain sorghum grits. Cereal Science Today, 1969. 14: p. 372-376.

[6] Bhattacharya, M., M.A. Hanna, and R.E. Kaufmann, Textural properties of extruded plant protein blends. JOURNAL OF FOOD SCIENCE, 1986. 51(4): p. 988-993.

[7] Konica-Minolta, SpectraMagic NX 2.03.0006, 2003-2010, Konica Minolta Sensing Inc.: Japan.

[8] Coulibaly, A., B. Kouakou, and J. Chen, Extruded Adult Breakfast Based on Millet and Soybean: Nutritional and Functional Qualities, Source of Low Glycemic Food. Journal of Nutrition \& Food Sciences, 2012. 2(7).

[9] Gamalth, S. and R. Ganesharanee, Extruded products with fenugreek (Trigonellagraecium) Chick pea and Rice: physical properties, sensory acceptability and glycemic index. Journal of Food Engineering, 2009. 90: p. 45-52.

[10] Deshpande, H.W. and A. Poshadri, Physical and sensory characteristics of extruded snacks prepared from Foxtail millet based composite flours. International Food Research Journal, 2011. 18: p. 730-735.
[11] Dobraszczyk, B.J., et al., Baking, Extrusion and Frying, in Food Processing Handbook, J.G. Brennan, Editor. 2006, Wiley-VCH Verlag GmbH \& Co. KGaA,: Weinheim, Germany. p. 237-290.

[12] Gomez, M.H., et al., Extrusion-cooking of sorghum containing different amount of amylose. JOURNAL OF FOOD SCIENCE, 1988. 53(6): p. 1818-1822.

[13] El-Dash, A.A., R. Gonzalas, and M. Ciol, Response surface methodology in the control of thermoplastic extrusion of starch, in Extrusion Cooking Technology, R. Jowitt, Editor. 1984, Elsevier Applied Science: London. p. 51.

[14] Guy, R.C.E., Raw Materials for Extrusion Cooking Processes, in The Technology of Extrusion Cooking, N.D. Frame, Editor. 1994, Blackie Academic and Professional: Glasgow. p. 52-72.

[15] Chinnaswamy, R. and M.A. Hanna, Expansion, color and shear strength properties of corn starches extrusion cooked with urea and salts. Starch/Stärke, 1988. 40: p. 186-190.

[16] Ilo, S. and E. Berghofer, Kinetics of color changes during extrusion cooking of maize grits. Journal of Food Engineering, 1999. 39: p. 73-80.

[17] Semasaka, C., X.Z. Kong, and Y. Hua, Optimization of extrusion on blend flour composed of corn, millet and soybean. Pakistan Journal of Nutrition, 2010. 9: p. 291-297.

[18] Guy, R., Extrusion cooking: technology and applications. 2001, Cambridge, England: Woodhead.

[19] Obatolu, V.A. and A.H. Cole, Functional property of complementary blends of soybean and cowpea with malted or unmalted maize. Food Chemistry, 2000. 70: p. 147-153.

[20] Filli, K.B., et al., Influence of of extrusion variables on some functional properties of extruded millet-soybean for the manufacture of 'fura': A Nigerian traditional food. African Journal of Food Science, 2010. 4(6): p. 342- 352.

[21] Thomas, D.J. and W.A. Atwell, Starches, in Eagan Press Handbook1997, Eagan Press: Minnesota, U. S. A.

[22] Jansen, G.R., et al., The Calorie densities of gruels made from extruded corn-soy blends. Food Nutrition Bulletin, 1981. 3(1): p. 39.

[23] Likimani, T.A., et al., Extrusion cooking of corn/soy bean mix is presence of thermostable a-amylase. JOURNAL OF FOOD SCIENCE, 1991. 56(1): p. 99-105.

[24] Arambula, V.G., et al., Milling and processing parameters for Corn Tortillas from Extruded instant Dry Masa flour. JOURNAL OF FOOD SCIENCE, 1998. 63(2): p. 338-341.

[25] Davidson, V.J., et al., Degradation of wheat Starch in a singlescrew extruder: Characteristics of extruded starch polymers. JOURNAL OF FOOD SCIENCE, 1984. 49: p. 453-458.

[26] Gomez, M.H. and J.M. Aguilera, Changes in the Starch Fraction During Extrusion-cooking of Corn. JOURNAL OF FOOD SCIENCE, 1983. 48: p. 378-381.

[27] Pelembe, L.A.M., C. Erasmus, and J.R.N. Taylor, Development of a Protein-rich Composite Sorghum-Cowpea Instant Porridge by Extrusion Cooking Process. LWT - Food Science and Technology, 2002. 35: p. 120-127. 